\title{
Im Sinne der Erhaltung des politischen und sozialen Friedens
}

\section{League of Nations and ILO - Guardians of Peace}

The Treaty of Versailles was signed on 28 June 1919, the same day on which the League of Nations was founded with the principal goals of safeguarding world peace and promoting international cooperation. The League of Nations Covenant, which was included in all of the Paris peace treaties, is regarded as the instrument for monitoring these treaties. The Covenant of the International Labour Organization (ILO) is also part of all Paris peace treaties - its primary aim is to promote universal social justice. Whereas the League failed in securing peace, the ILO continues to be an agency of the UN.

Keywords: Globalization - ILO - League of Nations - Peace - Treaty of St. Germain

Als am 28. Juni 1919 der Vertrag von Versailles unterzeichnet wurde, erfolgte gleichzeitig die Gründung des Völkerbundes (Teil I des Vertrages von Versailles) und der Internationalen Arbeitsorganisation (International Labour Organization, ILO, Teil XIII des Versailler Vertrages). Diese beiden internationalen Organisationen sind nicht nur Bestandteil des Versailler Vertrages, sondern aller Pariser Friedensverträge, wodurch die Verliererstaaten verpflichtet waren, sowohl den Völkerbund als auch die ILO anzuerkennen. In den folgenden Ausführungen werden Entstehungs- und Wirkungsgeschichte beider Organisationen in den Blick genommen und abschließend einer kritischen Analyse unterzogen.

\footnotetext{
${ }^{1}$ Dies ist die erweiterte Version des Beitrages von ZIEGERHOFER, League of Nations.

${ }^{2}$ BAUMGART, Vom europäischen Konzert 136. Allgemein zur Vorgeschichte (Auswahl): BARANDON, Völkerbund 597-611; GÖPPERT, Völkerbund; GOLDSTEIN, First World 35-37; HENIG, League of Nations; HousDEN, League of Nations 20-37; OsTROWER, League of http://dx.doi.org/10.1553/BRGOE2019-2s293
}

\section{Der Völkerbund ${ }^{1}$}

Der Völkerbund gilt als die erste internationale "globale" Organisation, die einerseits die Staaten der Welt zu einem strukturierten, institutionalisierten Dialog im Sinne der Erhaltung des politischen und sozialen Friedens zusammenführen und andererseits die Internationalisierung vorantreiben sollte. Seine Vorläufer reichen bis zur mittelalterlichen Vorstellung der christlichen Völkerwelt als eine geschlossene Völkerrechtsgemeinschaft „Res publica Christiana“ zurück, als deren oberster Schiedsrichter und Friedensverhandler der Papst vorgesehen war. ${ }^{2}$ Als am Beginn der Neuzeit diese Völkergemeinschaft in eine Anzahl eigenständiger Staaten zerfallen war, wurde diese christliche Vorstellung allmählich von der politischen Idee der „balance of

Nations; PARRY, League of Nations; BERNHARDT, Encyclopedia 177 (Zugriff 12. 12. 2018); PFEIL, Völkerbund 32-45; TAMS, League of Nations 2 (Zugriff 12. 12. 2018); ZIMMERN, League of Nations 228. 
power" verdrängt. ${ }^{3}$ Spätestens seit Abschluss des Westfälischen Friedens 1648 kam der Gedanke auf, dass internationale Organisationen ein Mittel sein könnten, unbeschränkte zwischenstaatliche Gewaltpolitik einzudämmen. ${ }^{4}$ In weiterer Folge ersannen Diplomaten, Philosophen oder Staatsmänner Ideen von einem europäischen Staatenbund, um einen Völkerfrieden zu erlangen. ${ }^{5} \mathrm{Zu}$ ihren prominentesten Vertretern, deren Visionen in der Völkerbundliteratur als Vorläufer des Völkerbundes genannt werden, zählen der Abbé de Saint-Pierre und Immanuel Kant, er verwendete erstmals das deutsche Wort Völkerbund in seinen Schriften. ${ }^{6}$ Der Wiener Kongress 1814 bildete einen wesentlichen Dreh- und Angelpunkt im tiefgehenden Strukturwandel der europäischen Politik, die sich vom Balance of Power-System des 18. Jahrhunderts zu einem Mächtegleichgewicht im „europäischen Konzert“ des 19. Jahrhunderts entwickelte. ${ }^{7}$ Mit diesem „europäischen Konzert", das am Wiener Kongress geschaffen wurde, errichtete man einen Prototyp einer internationalen Sicherheitsorganisation, die allerdings keine universale, globale Gültigkeit beanspruchte und bis zum Ausbruch des Ersten Weltkrieg bestand. ${ }^{8}$ Der Wiener Kongress etablierte auch ein Konsultativsystem von internationalen Treffen, deren Dynamik sich in den ab 1851 stattfindenden Weltausstellungen, Kongressen, Konferenzen und in der Gründung internationaler Organisationen niederschlug. ${ }^{9}$ So gelten die Jahre 1864 und 1865 als Annus mirabilis in der Geschichte der internationalen Organisationen, 1864 erfolgte die Gründung der Internationalen

\footnotetext{
${ }^{3}$ BAUMGART, Vom europäischen Konzert 137.

${ }^{4}$ RitTBERGER, ZANGL, KRUCK, Internationale Organisationen 50 .

${ }^{5}$ Vgl. allgemein zur Idee Europa BÖTTCHER, Klassiker des europäischen Denkens.

${ }^{6}$ Stellvertretend BARANDON, Völkerbund 597.

${ }^{7}$ Vgl. STAUBER, Wiener Kongress 245-246.

${ }^{8}$ RITTBERGER, ZANGL, KRUCK, Internationale Organisationen 50; STAUBER, Wiener Kongress 246.

${ }^{9}$ Herren, Internationale Organisationen 16-17.

${ }^{10}$ Ebd. 18-19.
}

Arbeiterassoziation in London, 1865 jene des Roten Kreuzes. Im selben Jahr schuf man mit der Internationalen Telegraphenunion erstmals den Typus einer internationalen Verwaltungsunion. ${ }^{10}$ So bestand am Vorabend des Ausbruchs des Ersten Weltkrieges ein weltweit verwobenes Netz an Staatenverbindungen, die auf internationalen Konventionen basierten. Die Friedensverträge von Paris dokumentieren diese Internationalisierung: So werden in Art. 234 des Vertrages von St. Germain allein 23 Übereinkommen und Verträge aufgezählt, zu deren Beitritt die Republik Österreich von den Alliierten und Assoziierten Mächten verpflichtet wurde. ${ }^{11}$ Schließlich trieb die internationale Friedensbewegung die Internationalisierung der politischen Ordnung beispielsweise in Form von Friedenskongressen voran. Unter dieser Perspektive sind beispielsweise die auf Anregung von Zar Nikolaus II. abgehaltenen Haager Friedenskonferenzen 1899 und 1907 zu verstehen: Sie führten zur Kodifizierung des humanitären Völkerrechts in der Haager Landkriegsordnung und zur Errichtung eines ständigen Schiedsgerichtes. ${ }^{12}$ Daran hatten private Institute wie das Institut de Droit International oder die International Law Association, beide 1873 gegründet, wesentlichen Anteil. ${ }^{13}$ Die Forderung der Pazifisten nach Abrüstung hingegen konnte nicht verwirklicht werden. ${ }^{14}$ Wenngleich sich bereits vor Ausbruch des Ersten Weltkrieges die Sichtweise über den Krieg geändert hatte, hielt dies die europäischen Mächte jedoch nicht davon ab, Krieg zu führen. ${ }^{15}$

${ }^{11}$ StGBl. 303/1920, Art. 234. Art. 234 entsprach dem Art. 282 Versailler Vertrag, dem Art. 217 Vertrag von Trianon, dem Art. 162 Vertrag von Neuilly und dem Art. 269 Vertrag von Sévres.

12 ZIMMERN, League of Nations 102-118.

${ }^{13}$ Vgl. dazu NeuHOLD, HuMMER, SCHREUER, Österreichisches Handbuch des Völkerrechts 1, 41.

${ }^{14}$ BAUMGART, Vom europäischen Konzert 137.

${ }^{15}$ MaCMillan, Die Friedensmacher 130. 
Während des Krieges rissen die Gründungen internationaler Organisationen und die Veranstaltung internationaler Kongresse nicht ab. ${ }^{16}$ Darüber hinaus begannen Staatsmänner der Entente, von neutralen Staaten und auch von Deutschland und Private, etwa die US-amerikanische „League to Enforce Peace“ oder die britische "League of Nations Society", 17 Entwürfe für die Schaffung eines Weltfriedensbundes $\mathrm{zu}$ verfassen. ${ }^{18}$ Unter ihnen befand sich der britische Blockademinister Robert Cecil, der 1916 seinen Plan „Proposals for the Maintenance of Future Peace" präsentierte. ${ }^{19}$ Weitere Ideen folgten u.a. von dem britischen Premierminister David Lloyd George, Frankreichs Premierminister Alexandre Ribot und von Woodrow Wilson. Letzterer gehört zu einem der einflussreichsten Verfechter eines weltumspannenden Friedensbundes. ${ }^{20}$ Schon einige Monate vor Eintritt der USA in den Weltkrieg hielt Wilson am 22. Jänner 1917 eine beachtliche Rede vor dem Senat, die Schwabe als „Schlüsselzeugnis für seine Friedenskonzeption" bezeichnete. ${ }^{21}$ Als die USA Anfang April 1917 dem Deutschen Reich den Krieg erklärten, betonte Wilson, Krieg gegen den Krieg führen und nicht europäische Gebiete erobern und „die Welt für die Demokratie sicher “22 machen zu wollen. Am 8. Jänner 1918 präsentierte er dem US-Kongress eine „14 PunkteErklärung", deren letzter Punkt die Vision von der Gründung einer allgemeinen Gemeinschaft von Nationen beinhaltete ,zum Zwecke wechselseitiger Garantieleistung für politische Unabhängigkeit und territoriale Unverletzlichkeit der großen wie auch der kleinen Staaten". ${ }^{23}$ Bald gründete man in England wie auch in Frankreich Regierungskommissionen unter dem Vorsitz von

\footnotetext{
${ }^{16}$ HERREN, Internationale Organisationen 52-53.

${ }^{17}$ MACMILLAN, Die Friedensmacher 135.

18 SCHÜCKING, WEHBERG, Satzung des Völkerbundes, auf den Seiten 6-10 werden diese Entwürfe aufgelistet.

${ }^{19}$ Allgemein YEARWOOD, Guarantee of peace.

${ }^{20}$ SCHÜCKING, WeHBERG, Die Satzung des Völkerbundes 5-10.

${ }^{21}$ SCHWABE, Weltmacht und Weltordnung 54.

22 Ebd. 59.
}

Walter Phillimore bzw. Léon Bourgeois; bereits im März präsentierte Phillimore ein erstes Ergebnis der Kommissionsarbeiten, Bourgeois lieferte im Juni seinen Erstbericht. ${ }^{24}$ Im Dezember 1918 veröffentlichte der südafrikanische General Jan Christian Smuts seinen Plan „The League of Nations. A Practical Suggestion“. ${ }^{25}$ Als Wilson im Dezember 1918 zur Friedenskonferenz aufbrach, hatte er bereits einen Entwurf für die Satzung des Völkerbundes im Reisegepäck, den er gemeinsam mit seinem engsten Mitarbeiter Edward House erarbeitet hatte.

Wilson ging in die Friedenskonferenz, die am 18. Jänner 1919 in Paris eröffnet wurde, mit der Forderung, nicht nur seinen Plan von einer Weltordnung durchsetzen, sondern die Völkerbundsatzung mit den Friedensverträgen, in erster Linie mit dem Versailler Vertrag, verbinden zu wollen. Dieses Junktim sollte sich letztlich als unheilvoll herausstellen und der Bedeutung des Völkerbundes einen enormen Imageschaden zufügen. Die Pariser Friedenskonferenz beauftragte am 20. Jänner den britischen Kronjuristen Cecil James Barrington Hurst und den amerikanischen Juristen David Hunter Miller mit der Ausarbeitung eines neuen Entwurfs, da der britische Regierungsentwurf sich vom Wilson-Entwurf wesentlich unterschied. Die beiden Juristen ließen die Ideen von Cecil, Smuts, House und Wilson in ihren Entwurf einfließen, der als Diskussionsgrundlage für die von der Pariser Friedenskonferenz eingesetzte Völkerbundkommission galt. ${ }^{26}$ Diese nahm Anfang Februar unter der Leitung von Wilson ihre Arbeiten auf, 11 Tage später prä-

\footnotetext{
${ }^{23}$ RAUMER, VierHAUS, Friede und Völkerordnung Teil II 31-33.

24 SCHÜCKING, WEHBERG, Satzung des Völkerbundes 6.

${ }^{25}$ Ebd. 11: Dort findet sich ein Überblick über die offiziellen und privaten Völkerbundentwürfe 6-10.

${ }^{26}$ Housden, League 33; SCHÜCKING, WEHBERG, Satzung des Völkerbundes 12-13.
} 
sentierte er bereits einen Entwurf der Völkerbundsatzung. ${ }^{27}$ Dann reiste Wilson in die USA zurück, um dort Werbung für seinen Plan zu machen. Hier sah er sich allerdings mit einer starken republikanischen Front gegen den Völkerbund konfrontiert - erste Vorzeichen der späteren Nichtratifizierung durch den US-Senat im März 1920. ${ }^{28}$ Nach Wilsons Rückkehr Anfang März und weiteren Diskussionen legte man der 5. Plenarsitzung der Friedenskonferenz den überarbeiteten Entwurf am 28. April 1919 vor. Wilson sah darin den Sieg seiner Prinzipien und meinte, dass mit der Völkerbundakte "etwas Lebendiges“ geboren wurde. Allerdings waren die Franzosen enttäuscht, da ihre Forderungen in Bezug auf Abrüstung und einer internationalen Polizeiorganisation niedergestimmt wurden, die Japaner darüber, dass man die Gleichberechtigung der „Rassen" nicht in die Satzung aufgenommen hatte und Australien und Südafrika fürchteten, dass sie die Mandatsaufteilung der deutschen Kolonien um ihren Kolonialbesitz bringen würde. Darüber hinaus hatten nun die Republikaner unter Henry Cabot Lodge ein „Dokument, auf das sie ihre Kritik konzentrieren konnten“ ${ }^{29}$

Wilson hatte die Unterzeichnung der Völkerbundstatuten mit jener des Versailler Vertrages junktimiert, daher gilt der 28. Juni 1919 als das offizielle Gründungsdatum der 26 Artikel umfassenden Satzung, erfolgte doch an diesem Tag die Unterzeichnung des deutschen Friedensvertrages. Die Völkerbundsatzung trat mit der gleichzeitigen Ratifizierung des Versailler Vertrages

\footnotetext{
${ }^{27}$ Vgl. SCHÜCKING, WEHBERG, Satzung des Völkerbundes 14 mit einer Auflistung der Kommissionsmitglieder; die Kommission begann am 3. Februar ihre Arbeit und endete mit 13. Februar 1919.

${ }^{28} \mathrm{Vgl}$. dazu den Beitrag von SCHWABE in diesem Band sowie LEONHARD, Überforderter Frieden 694-697.

${ }^{29}$ LEONHARD, Überforderter Frieden 701.

${ }^{30} \mathrm{Vgl}$. Ebd. 687-688.

${ }^{31}$ Ebd. 688.

32 StGB1. 303/1920.

33 StGB1. 303/1920 I. Ursprüngliche Mitglieder: Vereinigte Staaten von Amerika, Belgien, Bolivien, Brasilien,
}

am 10. Jänner 1920 in Kraft. Die presbyterianische Haltung des amerikanischen Präsidenten kam in der Wahl der Bezeichnung der Völkerbundsatzung als "Covenant" zum Ausdruck: Wilson wählte bewusst diesen auch religiös konnotierten Begriff, der sich auf den Bund Gottes mit den Menschen bezog. ${ }^{30}$ Die Völkerbundsatzung bildet den ersten Teil aller Friedensverträge - somit gilt der Völkerbund auch als Instrument ihrer Überwachung und mithin als Ausdruck einer „Universalisierung des amerikanischen Nationsverständnisses“. ${ }^{31}$

Das vorrangige Ziel des Weltareopags war die Gewährleistung des internationalen Friedens und der internationalen Sicherheit. Deshalb verpflichteten sich seine Mitgliedstaaten in der Präambel zu den Grundsätzen eines relativen, statt allgemeinen Kriegsverbotes, der Unversehrtheit des territorialen Besitzstandes, zu Gerechtigkeit in den internationalen Beziehungen, zur Bindung der Staaten an das Völkerrecht und zur Einhaltung vertraglicher Verpflichtungen. ${ }^{32}$ Die Satzung, die man durchaus als ein Hochdokument des Völkerrechts bezeichnen kann, gibt den Idealzustand wieder, doch sollte sich rasch zeigen, dass diese Idealvorstellung der Realität nicht standhalten konnte. Die Diskrepanz zwischen Ideal und Realität offenbarte sich bereits in der Frage der Mitgliedschaft. Insgesamt hätten die 32 „ursprünglichen“ Mitglieder (das waren die Vertragsunterzeichner der Friedensverträge) sowie 13 später hinzugekommene, 33 also 45 Mitglieder, den Völkerbund bilden sollen. Allerdings waren

Britisches Reich mit Canada, Australien, Südafrika, Neuseeland, Indien, China, Cuba, Ecuador, Frankreich, Griechenland, Guatemala, Haiti, Hedschas, Honduras, Italien, Japan, Liberia, Nicaragua, Panama, Peru, Polen, Portugal, Rumänien, Serbisch-KroatischSlowenischer Staat, Siam, Tschecho-Slowakei, Uruguay. Zum Beitritt zu der Satzung eingeladene Staaten: Argentinien, Chile, Kolumbien, Dänemark, Spanien, Norwegen, Paraguay, Niederlande, Persien, Salvador, Schweden, Schweiz, Venezuela. 
der Hedschas, ${ }^{34}$ Ecuador und die USA diesem nicht beigetreten. Nicht nur das Fernbleiben der USA und zunächst der UdSSR, sondern auch die vielen Austritte ${ }^{35}$ (insgesamt 17 Austritte bei 22 Beitritten) sollten den Völkerbund $\mathrm{zu}$ einem Torso machen und das System der Universalität untergraben: „The members failed the League rather than the League its members. "36 Übrigens, nur ein Mitglied wurde nach Art. 16 Abs. 4 ausgeschlossen: die UdSSR. ${ }^{37}$ Dem Völkerbund konnten nicht nur Staaten beitreten, sondern auch Kolonien und internationale Organisationen (Art. 24), deren Beitritt jedoch an die Zustimmung der Vertragsstaaten gebunden war. Da nicht alle Vertragsstaaten diesem Vorschlag zustimmten, waren bis 1939 nur sechs internationale Organisationen Mitglied des Völkerbundes - damit schuf man ,internationale Parallelwelten, die von unterschiedlichen Generationen internationaler Organisationen bevölkert waren. “38 Die Organisationsstruktur war einfach und schlank und bestand aus dem Rat, der Bundesversammlung und dem Sekretariat (Art. 2-6). Dem ständigen Sekretariat (Art.6), mit Sitz in Genf („Palais des Nations“), wurde die aktenmäBige Dokumentation und Führung der umfangreichen Verwaltungstätigkeiten überantwortet,

\footnotetext{
34 1924/26 von Saudi-Arabien erobert.

${ }^{35}$ KÖCK, FISCHER, Recht der Internationalen Organisationen 148: Unter den Staaten, die austraten, befanden sich z.B. Japan 1933, Deutschland 1933 und Italien 1937; Österreich und Albanien schieden aus, als sie ihre staatliche Souveränität verloren hatten.

36 PARRY, League of Nations; BERNHARDT, Encyclopedia of public International Law III 185.

${ }^{37}$ KÖCK, FISCHER, Recht der Internationalen Organisationen 148: Die Sowjetunion trat 1934 dem Völkerbund bei, wurde aber aufgrund des Angriffs auf Finnland 1939 ausgeschlossen.

${ }^{38}$ HerREN, Internationale Organisationen 60-61. Diese internationalen Organisationen waren das Central International Office for the Control of the Liquor Traffic in Africa (Sitz: Brüssel), Internationale Hydrographic Bureau in Monaco, International Exhibition Bureau in Paris (zur Organisation der Weltausstellung 1928 gegründet), International Commission for Air Navigation in Paris, Nansen International Office for Refugees
}

es bildete das „Gedächtnis des Völkerbundes“.39 Das Sekretariat des Völkerbundes hatte in den 1930er Jahren 700 Mitarbeiter, wovon der Großteil der Mitglieder EuropäerInnen waren. ${ }^{40}$ Als erster Generalsekretär fungierte der Brite Sir Eric Drummond, gefolgt von dem französischen Diplomat Joseph Avenol, der letzte Generalsekretär war Seán Lester, früherer Hoher Kommissar in Danzig.41 Präsident des Völkerbundes wurde 1923 Sir Robert Cecil, er blieb in dieser Funktion bis 1946, 1937 erhielt er den Friedensnobelpreis. Der Ständige Internationale Gerichtshof war kein Völkerbundorgan, seine Gründung war in Art. 14 nur vorgesehen. ${ }^{42}$

Nicht nur das Fernbleiben der USA vom Völkerbund erwies sich als gewaltiger Geburtsfehler, sondern auch die Tatsache, dass sich der Völkerbund gegen die interalliierten Einrichtungen, das waren der „Oberste Rat“ und die „Botschafterkonferenz" nur schwer durchsetzen konnte. ${ }^{43}$ So hatte man auch zur Lösung der Reparationskosten, die einen wesentlichen Teil der Friedensverträge ausmachten, eine interalliierte Reparationskommission unter französischer Leitung geschaffen, ${ }^{44}$ der Völkerbund konnte lediglich finanzielle und wirtschaftliche Sanierungspläne, so etwa für

in Genf und das International Office for Information and Research Concerning Assistance to Foreigners in Paris. Der Weltpostverein trat dem Völkerbund nicht bei.

39 BARANDON, Völkerbund 602.

${ }^{40}$ ROSENBERG, Transnationale Strömungen 843.

${ }^{41}$ BARANDON, Völkerbund 602.

42 KÖCK, FISCHER, Recht der Internationalen Organisationen 157: Der Rat war mit dem Entwurf der Statuten des Ständigen Internationalen Gerichtshofes betraut; dieser wurde nach Überarbeitung am 16. Dezember 1920 von den Völkerbundmitgliedern unterzeichnet. Im Herbst 1921 nahm er seine Arbeit auf mit Sitz in Den Haag vgl. RAUMER, VIERHAUS, Friede und Völkerordnung II, 41-44.

${ }^{43}$ HeideKING, Oberster Rat 589-630; Vgl. auch den Beitrag RATHMANNER in diesem Band.

${ }^{44}$ Vgl. dazu RATHMANNER, Reparationskommission 81-96. 
Österreich und Ungarn, ausarbeiten..$^{45}$ Im Zusammenhang mit den Reparationskosten erhofften sich die besiegten Staaten eine rasche Änderung der Friedensverträge und bezogen sich dabei auf Art. 19 der Völkerbundsatzung. Dieser Artikel bestimmte, dass die Völkerbundversammlung das Recht zur Revision einiger Artikel der Friedensverträge „deren Aufrechterhaltung den Weltfrieden gefährden könnten“, besaß. ${ }^{46}$ Dieser "Revisionsartikel“" galt auch als Hoffnung für die revisionistischen Wünsche, ein Erfolg auf Revision war diesen Staaten jedoch nicht beschieden, da ein diesbezüglicher Beschluss von der Bundesversammlung einstimmig zu fällen war. ${ }^{47}$

Die politische Hauptaufgabe des Völkerbundes bildete die internationale institutionelle Friedenssicherung, fußend auf dem Prinzip der friedlichen Streitbeilegung und der kollektiven Sicherheit. Als Voraussetzung dafür galt die Abrüstung, wie sie Wilson auch im vierten Punkt seiner Erklärung von 1918 vorgeschlagen hatte. So waren alle Bundesmitglieder zur Rüstungsbeschränkung „,auf das Mindestmaß, [...] das mit der nationalen Sicherheit und mit der Erzwingung internationaler Verpflichtungen durch gemeinschaftliches Vorgehen vereinbar ist" ${ }^{48}$ verpflichtet. Der Rat sollte mit Hilfe des Rüstungsausschusses Abrüstungspläne entwerfen. Bereits sein erster Versuch, der sich in der Schaffung des Genfer Protokolls vom 2. Oktober 1924 niederschlug, scheiterte am Widerstand von Großbritannien. ${ }^{49}$ Für die damaligen Völkerbundkritiker, darunter der Gründer der Paneuropa-Union

\footnotetext{
${ }^{45}$ FreUnD, Genfer Protokolle sowie vgl. dazu Walter IBER, in diesem Band.

${ }^{46}$ StGBl. 303/1920, Art. 19 Völkerbundsatzung.

47 BARANDON, Völkerbund 303.

48 StGBl. 303/1920, Art. 8 Völkerbundsatzung.

${ }^{49}$ Vgl. dazu auch IPSEN, Völkerrecht 1060: Das Genfer Protokoll enthielt ein Verbot des Angriffskrieges, die Verpflichtung zum Verteidigungs- und Sanktionskrieg im Rahmen eines Systems kollektiver Sicherheit und die Verpflichtung zur friedlichen Streitbeilegung und Rüstungsbeschränkung.
}

Richard Nikolaus Coudenhove-Kalergi, bedeutete dieses Scheitern von 1924 bereits das Ende des Völkerbundes. ${ }^{50}$

Als Folge der gescheiterten Protokolle setzte der Rat 1925 eine Kommission ein, die eine internationale Abrüstungskonferenz vorbereiten sollte. Diese „Internationale Konferenz zur Herabsetzung und Begrenzung von Rüstungen" wurde am 2. Februar 1932 in Genf eröffnet aufgrund mangelnden Erfolgs Ende Juli unterbrochen, um sie im Februar 1933 wieder fortzusetzen. ${ }^{51}$ Im Jänner zuvor war Hitler in Deutschland Reichskanzler geworden, seine Pläne zur Wiederaufrüstung standen diametral zu jenen der Konferenz, da half auch der Plan des britischen Premiers MacDonald nicht mehr, weshalb Hitler am 14. Oktober 1933 nicht nur die Abrüstungskonferenz verließ, sondern auch den Austritt des Deutschen Reichs aus dem Völkerbund erklärte. ${ }^{52}$ Damit waren sämtliche Bemühungen, dem Krieg durch Abrüstung die materiellen Grundlagen zu entziehen, gescheitert. Im Gegensatz zum Völkerbund waren diesbezügliche Initiativen einzelner Völkerbundmitglieder zunächst erfolgreich: So unterzeichneten die Alliierten und Assoziierten Mächte im Februar 1922 im Zuge der Washingtoner Abrüstungskonferenz eine Vereinbarung über Seerüstung, die durch das Londoner Protokoll 1930 eine Bestätigung erfuhr, allerdings 1936 auslief. ${ }^{53}$

Einen gewaltigeren Imageverlust erfuhr der Völkerbund jedoch im Laufe der 1930er Jahre: Die ab jener Zeit sich häufenden internationalen Konflikte $^{54}$ und schließlich der Abessinienkrieg55

\footnotetext{
${ }^{50}$ Coudenhove-KalergI, Pan-Europa 3-23 sowie DERS., Weltpolitik 32-37.

${ }^{51}$ PfEIL, Völkerbund 101-120.

52 KERSHAW, Hitler 623-625.

53 HeISER, Abrüstung 5ff.

${ }^{54}$ Vgl dazu PfEIL, Völkerbund, Die Phase der Konflikte 1931-1936, 103-136 oder ZIMMERN, League of Nations 404-458; HOUDSEN, League 93-110.

${ }^{55}$ Ebd. 102-106; ferner THÖNDL, Mussolini 449-488.
} 
führten der Weltöffentlichkeit die Schwäche des Völkerbundes drastisch vor Augen: Das in Art. 10 (Angriffsverbot und Unversehrtheit des Gebietes) ${ }^{56}$ und Art. 16 (Sanktionen) explizit erwähnte Prinzip der „kollektiven Sicherheit“ war $\mathrm{zu}$ einer Farce geworden. Die wirtschaftlichen Sanktionen gegen Italien griffen zu kurz, zu militärischen Sanktionen waren die Mitgliedstaaten nicht verpflichtet. Übrigens erfuhr Art. 10 durch den Locarno-Pakt eine Ergänzung, indem die Signatarstaaten die deutsch-belgische bzw. deutsch-französische Grenze, wie sie im Versailler Vertrag gezogen worden war, garantierten. Die Ratifizierung des Locarno-Vertragswerkes bildete die Voraussetzung für Deutschland, dem Völkerbund beitreten zu können, was 1926 geschah. Das Jahr 1936 läutete schließlich die Agonie des Völkerbundes ein.

Der Völkerbund versagte nicht nur in seinen "Herzbereichen" kollektive Sicherheit und Abrüstung, sondern auch in der friedlichen Streitbeilegung (Art. 15). Schließlich waren wiederum Initiativen einiger Staatsmänner in Bezug auf „Kriegsverbot" vorerst scheinbar erfolgreicher als jene des Völkerbunds. Dieser kannte lediglich ein relatives Kriegsverbot - dies ließ den Rückschluss zu, dass ein legaler Krieg gerechtfertigt war, und zwar nach Ablauf bestimmter Fristen und gewisser Bedingungen. So enthielt der Locarno-Pakt, der am 1. Dezember 1926 in London unterzeichnet wurde, ein Kriegsverbot und der am 27. August 1928 geschlossene Briand-Kellogg-Pakt (Kriegsächtungsplan) ächtete den Krieg als ultima ratio für die Lösung internatio-

\footnotetext{
${ }^{56}$ Die Frage der kollektiven Sicherheit wird mit Art. 10 geregelt. Die Mitglieder des Völkerbundes verpflichteten sich dazu, die territoriale Unversehrtheit und politische Unabhängigkeit aller Bundesmitglieder zu garantieren und betrauten den Rat, bei Nichterfüllung dieser Verpflichtungen dementsprechende Maßnahmen zu ergreifen. Durch diesen Besitzschutz wurde der territoriale Status quo von 1920 gesichert. Der Inhalt des Art. 10 wurde von den „imperialistischen“ und „isolationistischen“ Kritikern als Eingriff in die
}

naler Streitfälle. ${ }^{57}$ Allerdings fehlten diesem multilateralen Vertragswerk Mechanismen zur Durchsetzung und zum Schuldnachweis. ${ }^{58}$

Wie bereits erwähnt kannte der Völkerbund kein allgemeines Kriegsverbot. So bestimmte Art. 11, dass jeder Krieg und jede Kriegsdrohung eine Bundesangelegenheit war. In den Anfangszeiten des Völkerbundes beriefen sich die Mitgliedstaaten bei nahezu allen Streitfällen auf diesen Artikel. Satzungsgemäß waren die Bundesmitglieder zur friedlichen Streitbeilegung verpflichtet, die auf drei Wegen erreicht werden konnte: Schiedsgerichtsbarkeit, gerichtliches Verfahren oder Prüfung durch den Rat (Art. 12). Art. 13 präzisierte diese Streitfragen, sie bezogen sich auf Fragen über Vertragsauslegung, das internationale Recht bzw. Verletzung das internationale Recht betreffend (Art. 13 Abs. 2). Die Bundesmitglieder waren verpflichtet, die Schiedssprüche zu befolgen und gegen keinen Staat, der sich einer schiedsgerichtlichen Entscheidung unterwarf, Krieg zu führen. Streitfragen mussten entweder einem ad hoc vereinbarten Gericht oder dem in Art. 14 vorgesehenen und 1920 in Den Haag gegründeten Ständigen Internationalen Gerichtshof vorgelegt werden. Art. 13 bildete eine lex imperfecta, einzelne Völkerbundmitgliedstaaten schlossen untereinander Schiedsgerichts- oder Vergleichsverträge wie z.B. den Locarno-Pakt. ${ }^{59}$ Schließlich legte Art. 15 das aufwendige Vermittlungsverfahren fest. Zu den wenigen Art. 15-Verfahren zählte etwa der Mandschurei-Konflikt (1931-1933), auch Haile Selassie bezog sich, allerdings ohne Erfolg, auf diesen Artikel am Vorabend des Abessinienkrieges. Zieht man Bilanz

Souveränitätsrechte Amerikas angesehen und daher abgelehnt, siehe dazu SCHWABE, Weltmacht 73-75.

${ }^{57}$ WeHBerg, Briand-Kellogg-Pakt 249ff; PfeIL, Völkerbund 91 sowie HUBER, Deutsche Verfassungsgeschichte 557-562 sowie 572-573.

${ }^{58}$ ROSENBERG, Transnationale Strömungen 846.

${ }^{59}$ KÖCK, FISCHER, Recht der Internationalen Organisationen 174. 
über die Streitfälle, die im Zeitraum von 1921 und 1936 an den Rat herangetragen wurden, so konnte dieser von insgesamt 24 Fällen lediglich in fünf erfolgreich vermitteln und zwar ausschließlich bei Streitigkeiten zwischen Klein- und Mittelstaaten, sechs Fälle blieben unerledigt, bei Streitigkeiten unter den Großmächten war er ebenfalls erfolglos. 60

Aus den erwähnten Artikeln ist zu schließen, dass ein legaler Krieg nach Ablauf bestimmter Fristen, im Falle der Selbsthilfe gegen eine widerspenstige Partei oder bei gescheitertem Vermittlungsverfahren mangels einstimmigen Ratsbeschlusses zulässig war. In den Fällen des legalen Krieges waren sowohl die angegriffenen Staaten wie auch alle Bundesmitglieder in ihren Entscheidungen frei, d.h. neben der Selbsthilfe war auch die Hilfeleistung legal Kriegführender erlaubt. ${ }^{61}$ Im Zuge der Pariser Friedensverhandlungen diskutierte man die Frage der Kolonien. Jan Christian Smuts hatte in seinem Völkerbund-Entwurf von Dezember 1918 die Idee des Mandatssystems aufgeworfen, er wollte in erster Linie die erdölreichen Gebiete im Nahen Osten unter internationale Kontrolle stellen. Diese Idee stand jener von Wilson entgegen, der von einem Frieden ohne „Besetzung" ausging. 62 Schließlich einigte man sich darauf, das Mandatssystem dem Völkerbund $\mathrm{zu}$ überantworten, diese Einigung findet sich in Art. 22 seiner Satzung. Der Völkerbund verfolgte in dieser Frage im Sinne von Wilsons fünften Punkt eine „zivilisatorische Mission“: „Das Wohlergehen und die Entwicklung

${ }^{60}$ FRIEDMANN, Vom Völkerbund zur UNO 13.

${ }^{61}$ BARANDON, Völkerbund 606.

62 Pedersen, Mandates System 560.

63 StGBl. 303/1920, Art. 22 Völkerbundsatzung.

${ }^{64}$ Art. 22 nennt weder die Mandatsmächte noch die Mandatsvergabe. Diese erfolgte vom alliierten Obersten Rat, siehe die Auflistung bei PFEIL, Völkerbund 5960: A-Mandate (ehemals türkische Gebiete) waren Syrien und Libanon (Frankreich), Palästina und Transjordanien (Großbritannien), Mesopotamien (Irak) (Großbritannien). B-Mandate (ehemals deutsche Schutzgebiete): Togo und Kamerun (geteilt) (Frankreich und Großbritannien), Deutsch-Ostafrika (Tanganyika) dieser Völker“ empfand er als „eine heilige Aufgabe der Zivilisation“, weshalb Wilson den „fortgeschrittenen Nationen" die Vormundschaft über jene Völker übertrug, „, die infolge des Krieges aufgehört haben, unter der Souveränität der Staaten $\mathrm{zu}$ stehen, die sie vorher beherrschten [...], die noch nicht imstande sind [...] sich selbst zu leiten."63

Bei der Aufteilung der Kolonien handelte es sich um die deutschen Kolonien und die arabischen Gebiete des Omanischen Reiches, die vom „Obersten Rat" in drei unterschiedliche Mandate eingeteilt wurden. ${ }^{64}$ Großbritannien und Frankreich erhielten den größten Anteil an Mandaten, Japan, Südafrika, Australien, Neuseeland und Belgien einen kleineren Teil, und Italien ging leer aus. ${ }^{65}$ Auf der Pariser Friedenskonferenz diskutierte man die armenische Frage und war auf der Suche nach einem Mandatar. Allerdings weigerten sich sowohl die europäischen Mächte wie auch die USA. Der Völkerbund begründete seine Absage damit, weder Mittel noch Truppen zu besitzen, um Armeniens Integrität gewährleisten zu können. ${ }^{66}$ Diese Gebiete sollten von „fortschrittlichen Nationen" verwaltet werden, die Mandatsverwalter waren laut Statuten zu einem Mindestmaß an Menschenrechten verpflichtet: zur gerechten Behandlung der "eingeborenen“ (sic!) Bevölkerung und "Entwicklung des Landes". ${ }^{67}$ Das Mandatssystem wurde von einer „Ständigen Mandatskommission" verwaltet, die sich auf die Mithilfe einer Sektion im Völkerbundsekretariat stützen konnte. Ihr Sitz war in Genf, das erste

(Großbritannien), Deutsch Ostafrika (Ruanda-Urundi) (Belgien) C-Mandate (ehemals deutsche Schutzgebiete); Deutsch-Südwestafrika (Südafrikanische Union), Westsamoa (Neuseeland)

Nauru (Australien), Neuguinea und Besitzungen im Pazifik südlich des Äquators (Australien), Besitzungen im Pazifik nördlich des Äquators (Australien).

${ }^{65}$ KÖCK, FISCHER, Recht der Internationalen Organisationen 187.

${ }^{66} \mathrm{Vgl}$. BANKEN, Verträge von Sévres 219-221.

${ }^{67}$ StGBl. 303/1920, Art. 22 Völkerbundsatzung. 
Treffen der Kommission fand am 4. Oktober 1921 statt. ${ }^{68}$ Dieses System sollte mit der Unabhängigkeit jener Gebiete enden, wobei lediglich der Irak 1932 und der Libanon 1943 diese erhielten. Der Völkerbund stützte den Kolonialismus, indem das Mandatssystem die Fremdverwaltung sanktionierte und legitimierte, die Kolonialvölker, die auf Wilsons gefordertes Selbstbestimmungsrecht so sehr vertrauten, wurden bitter enttäuscht. Aus ihrer Sicht integrierte der Völkerbund den Kolonialismus in einen eurozentrierten Internationalismus. ${ }^{69}$ Das Selbstbestimmungsrecht wurde zu einer zentralen Komponente der Nationalisten, um den Imperialismus in internationale Beziehungen umzuwandeln. Dennoch brachte "the Wilsonian Moment" mehr Konflikte als Zusammenarbeit. ${ }^{70}$

Hinsichtlich Minderheitenrechten beinhaltete die Satzung keine Bestimmung, sie waren in den Minderheitenschutzverträgen zu den Friedensverträgen verankert. ${ }^{71}$ Der Völkerbund sah sich als (erfolgloser) Garant für die Minderheitenrechte, da er über die Friedensverträge zu wachen hatte. Seine Minderheitenpolitik kann theoretisch als konstruktiv und friedensfördernd bezeichnet werden, scheiterte aber am Verhalten der Staaten. ${ }^{72}$ Der Ständige Internationale Gerichtshof war sowohl für diesbezügliche Streitigkeiten als auch jene in Bezug auf Mandatsbereiche zuständig.

\section{Die Internationale Arbeitsorganisation (ILO)}

Der Völkerbund verstand sich nicht nur als internationale Organisation zur Erhaltung des politischen Friedens, sondern auch des sozialen Friedens. Darüber hinaus sah er sich als Motor zur „Förderung der Zusammenarbeit unter den Nationen" ${ }^{73}$ In dieser Funktion war er erfolgreicher als in seiner selbstauferlegten Rolle eines universellen politischen Friedensvermittlers. Zum Zwecke der „Förderung der Zusammenarbeit der Nationen" verfolgte er den Plan, alle bereits bestehenden oder künftig zu schließenden internationalen Übereinkommen ihm zu unterstellen und verpflichtete sich, dementsprechende Standards wie etwa angemessene Arbeitsbedingungen oder gerechte Behandlung der „eingeborenen“ Bevölkerung zu sichern, sowie den Handel von Mädchen und Kindern, Opium oder Waffen zu überwachen (Art. 23). Wenngleich es dem Völkerbund nicht gelang, die bestehenden Organisationen unter seinem Dach zu vereinen, konnte er gemäß Art. 24 neue Organisationen gründen. Eine davon ist die ILO, die erste Teilorganisation des Völkerbundes. In Art. 23 seiner Satzung verpflichtete der Völkerbund seine Mitglieder zu Mindeststandards von Arbeiternehmerrechten.

Die ILO ist wie der Völkerbund Teil der Friedensverträge. ${ }^{74} \mathrm{Ihr}$ Gründungsdokument ist demnach der Vertrag von Versailles, der erste Vertrag, der in Paris unterzeichnet wurde und der im Teil XIII (Art. 387-427) die organisatorischen und programmatischen Grundlagen beinhaltet. Ihre Gründung, die am 28. Juni 1919 erfolgte, war von der Überzeugung getragen, dass in der Förde-

au/other/dfat/treaties/1920/14.html (12. 12. 2018). PRITCHARD, Minderheitenschutz oder VIEFHAUS, Minderheitenfrage.

72 PoEgGEL, Völkerbund 22.

${ }^{73}$ StGBl. 303/1920, Präambel.

${ }^{74}$ StGBl. 303/1920, Teil XIII Arbeit Art. 332-372; Vertrag von Versailles Art. 387-427; Vertrag von

\footnotetext{
68 Pedersen, Meaning 560.

${ }^{69}$ RosenberG, Transnationale Strömungen 845.

${ }^{70}$ MANELA, Wilsonian Moment 218-219.

${ }^{71}$ Zum Beispiel Minderheitenvertrag Österreich mit der CSR: http://www.austlii.edu.au/au/other/dfat/ treaties/1920/5.html oder Minderheitenvertrag Österreich mit dem SHS-Staat: http://www.austlii.edu.au/
} 
rung der sozialen Gerechtigkeit durch Verbesserung der Arbeits - und Lebensbedingungen die notwendige Voraussetzung für einen dauerhaften Frieden läge. Sozialen Frieden betrachtete man als Garant und Gewährleistung für den Weltfrieden - das war ein neues Verständnis von Frieden. ${ }^{75}$ Die Motoren für die Gründung der ILO waren der Krieg und die russische Revolution.

Die Ursprünge der ILO reichen bis zum Beginn des 19. Jahrhunderts zurück, die diesbezüglichen Initiativen Privater entstanden vor dem Hintergrund der Industriellen Revolution und deren Auswirkungen auf die Gesellschaft. ${ }^{76}$ Der wohl einflussreichste Pionier der ILO war der Elsäßer Industrielle Daniel Legrand. ${ }^{77}$ Er verschickte in der Zeit von 1838 bis 1859 Denkschriften an Parlamente und Regierungen in Frankreich, England, Russland und der Schweiz mit der Forderung, einzelstaatliche und internationale Gesetze zum Schutz der Arbeiterschaft ausarbeiten zu wollen. Wesentlichen Einfluss übte die erste Internationale Arbeiterassoziation aus, die 1864 in London gegründet wurde: Sie trieb mit ihren Forderungen wie z.B. Verbot der Kinderarbeit unter 14 Jahren, generelles Nachtarbeitsverbot für Kinder, 8 Stunden-Tag, ein freier Tag pro Woche, die Schaffung eines internationalen Mindestlohnes und eines Systems von nationaler und internationaler Arbeitsinspektion die internationale Arbeitsschutzgesetzgebung voran. ${ }^{78} \mathrm{Ab}$ den 1890er Jahren organisierten Arbeitsreformer, vielfach auf Schweizer Initiative, einschlägige Konferenzen, die einerseits 1900 zur Gründung der Internationalen Vereinigung für Arbeitsrecht (International Association for Labour Legislation

Trianon Art.315-355; Vertrag von Neuilly Art. 249289; Vertrag von Sévres Art. 374-414.

75 Vgl. dazu LEONHARD, Überforderter Frieden 703.

${ }^{76}$ Vgl. ECKHARDT, KUTTIG, Arbeitsrecht im Friedensvertrage 1-7 oder Internationales Arbeitsamt, Zehn Jahre Internationale Arbeitsorganisation.

77 JOHNSTON, International Labour Organisation 5ff. sowie MACHACEK, Internationale Arbeitsorganisation 754 .
IALL) und andererseits schließlich zur Gründung der Vorläuferorganisation der ILO führten: das war das Internationale Arbeitsamt, gegründet am 1. Mai 1901 in Basel. ${ }^{99}$ Erster Präsident wurde der Basler Universitätsprofessor Stephan Bauer. Ende 1918 waren 23 Regierungen im Internationalen Arbeitsamt vertreten, mit vielen weiteren Regierungen stand es in Verbindung, sodass man es sogar als „,sozialpolitischen Völkerbund" bezeichnete. 80 Der Ausbruch des Ersten Weltkrieges ließ alle diesbezüglichen Bemühungen zunächst ruhen, allerdings organisierten Arbeiternehmervertreter der alliierten und neutralen Länder sowie der Zentralmächte ab 1916 bis 1918 Konferenzen und forderten von den Regierungen dementsprechende Maßnahmen in Bezug auf das Recht auf Arbeit, das Recht auf berufliche Vereinigung, Sozialversicherung, Arbeitszeitregelungen, Unfallverhütung etc. Ihre Forderungen sollten Gegenstand der bevorstehenden Friedensverhandlungen werden.

Nicht nur der Druck der internationalen Arbeiterschaft auf die Friedenskonferenz war groß und forderte rasches Handeln, sondern auch der Ausbruch der Oktoberrevolution 1917. So erklärt sich die Tatsache, dass die Pariser Friedenskonferenz bereits am 25. Jänner 1919 die Einsetzung eines Ausschusses von 15 Mitgliedern beschloss, der sich der Beschäftigungsverhältnisse von Arbeitnehmern aus internationaler Perspektive annehmen sollte mit dem Ziel, dem Frieden „,seine volle Bedeutung dadurch zu geben, dass sie durch internationale Übereinkommen die Würde und Freiheit der menschlichen Arbeit schütze“ ${ }^{.81}$ Die Arbeiten der Kommission begannen unter dem

\footnotetext{
78 JOHNSTON, International Labour Organisation 10-11.

${ }^{79} \mathrm{KUNZ}$, Internationales Arbeitsrecht 43.

${ }^{80}$ Ebd.

${ }^{81}$ OLIVETTI, Zehn Jahre Tätigkeit 15-16.
} 
Vorsitz des amerikanischen Arbeiterführers Samuel Gompers am 1. Februar und endeten - nach 35 Sitzungen - am 24. März 1919. ${ }^{82}$ Weitere Mitglieder dieser "Commission on Labour Regulation" waren bekannte Sozialpolitiker und Gewerkschafter wie George Barnes, Èmile Vandervelde oder Léon Jouhaux. ${ }^{83}$ Das schnelle Erarbeiten von Statuten ist auch den politischen Ereignissen in Ungarn und Bayern geschuldet: Hier versuchte man im März und April Räterepubliken zu installieren. Mittels ILO wollte man soziale Revolutionen vorab im Keim ersticken, weshalb Sozialisten und Gewerkschafter davon überzeugt waren, dass die Aufnahme sozialpolitischer Standards in die Völkerbundakte und schließlich in die Friedensverträge, diese legitimiere und man dadurch die Nachkriegsgesellschaft stabilisieren könne. ${ }^{84}$

Der Kommissionsbericht basierte auf zwei Papieren. Papier eins stellte die Statuten der ILO dar, Papier zwei beinhaltete allgemeine Grundsätze. Beide Papiere wurden nach kurzen Diskussionen angenommen. Gleichzeitig mit der Unterzeichnung des Versailler Vertrages und der Völkerbundsatzung erfolgte die Gründung der ILO am 28. Juni 1919 in Versailles unter Schirmherrschaft des Völkerbundes. ${ }^{85}$ Demnach waren alle Völkerbund-Signatarstaaten Mitglied der ILO, allerdings wurde bereits in den frühen 1920er Jahren die Frage der verpflichtenden Völkerbundmitgliedschaft als Voraussetzung für die Mitgliedschaft bei der ILO angezweifelt. Abgesehen von Deutschland, das damals noch nicht Völkerbundmitglied werden konnte, aber als Mitglied in die ILO bereits 1919 aufgenommen wurde, bot El Salvador den konkreten Anlassfall, als es als NichtVölkerbundmitglied Anfang der 1920er Jahre die

\footnotetext{
82 JOHNSTON, International Labour Organisation 13.

${ }^{83}$ KUNZ, Internationales Arbeitsrecht 48: Neben den erwähnten Vertretern aus den USA, Großbritannien, Frankreich und Belgien waren auch Vertreter aus Japan, Kuba, Polen und der Tschechoslowakei anwesend.

${ }^{84}$ LEONHARD, Überforderter Frieden 705.

85 Ebd. 3
}

Mitgliedschaft bei der ILO beantragte, ohne einen Pariser Friedensvertrag unterzeichnet zu haben. El Salvador wurde 1919 Mitglied der ILO und 1924 des Völkerbundes. Bald stellte sich auch die Frage, ob jene Staaten, die aus dem Völkerbund ausgetreten waren, weiterhin ILO-Mitglied bleiben konnten. 1928 hatte Brasilien den Völkerbund verlassen, der Generalsekretär des Völkerbundes und der Direktor der ILO entschieden sich für einen Verbleib. 86 So stand dem Ansuchen des US-amerikanischen Präsidenten Theodore Roosevelt um Beitritt zur ILO im Jahr 1934 nichts im Wege. Die Vereinigten Staaten von Amerika wurden ILO-Mitglied ohne dem Völkerbund beigetreten $\mathrm{zu}$ sein, dieser speziellen Vorgehensweise war allerdings ein relativ komplexes Verfahren vorangegangen. ${ }^{87}$ Bis 1936 traten Mexiko (1931), Irak und Türkei (1932), USA, Afghanistan, Ecuador und die UdSSR (1934) sowie Ägypten (1936) der ILO bei, bis auf die USA und Ägypten waren alle anderen Staaten aufgrund ihrer Völkerbundmitgliedschaft Mitglied der ILO geworden. Ab 1935 begann die Periode des Ausstieges, die bis 1942 währte: Deutschland (1935), Paraguay (1937), Österreich, Guatemala, Honduras und Nicaragua (1938), Italien und El Salvador (1939), schließlich Japan und die UdSSR (1940), Spanien (1941) und Rumänien (1942). 1942 verzeichnete die ILO den absoluten Tiefpunkt an Mitgliedern. Erst 1944 sollte es zu einem Anstieg der Mitglieder kommen. ${ }^{88}$

Die Statuten der ILO umfassen eine Präambel und 39 Artikel, sie bilden den XIII. Teil aller Friedensverträge. ${ }^{89}$ In der Präambel verwies man u.a. auf die ungerechten und schlechten Arbeitsbedingungen für eine große Anzahl von Menschen,

\footnotetext{
${ }^{86}$ JOHNSTON, International Labour Organisation 18.

87 Ebd. 19.

${ }^{88}$ Ebd. 21.

${ }^{89} \mathrm{Im}$ Folgenden werden die jeweiligen Artikel der ILO basierend auf den Vertrag von St. Germain zitiert.
} 
weshalb man eine Arbeitszeitregelung, die Festsetzung einer Höchstdauer für Arbeitstag und Arbeitswoche forderte sowie die Regelung des Arbeitsmarktes, Maßnahmen gegen Arbeitslosigkeit, angemessene Löhne, die angemessene Lebensbedingungen gewährleisten, weiters den Schutz der Arbeiter gegen Berufskrankheiten und Arbeitsunfälle, den Schutz von Kindern, Jugendlichen und Frauen, eine Alters - und Invalidenunterstützung, den Schutz der Interessen der im Ausland beschäftigten Arbeiter, die Anerkennung des Grundsatzes der Freiheit gewerkschaftlichen Zusammenschlusses und die Gestaltung des beruflichen und technischen Unterrichts..$^{90}$

Die ILO wurde als ständiger Verband gegründet. Ihre beiden Organe, Hauptversammlung (Art. 234) und Internationales Arbeitsamt (Art. 237), waren je mit Regierungsvertretern auf der einen sowie Arbeiternehmer- und Arbeitergebervertretern auf der anderen Seite besetzt. Dieser Tripartismus unterscheidet die ILO von anderen internationalen Organisationen, sie ist die einzige internationale zwischenstaatliche Organisation, in der die Regierungen kein alleiniges Stimmrecht besitzen, um internationale Standards zu setzen - Arbeitnehmer- und Arbeitgebervertreter haben das gleiche Recht wie die Regierungen! ${ }^{11}$ Sowohl Arbeitnehmervertreter als auch Arbeitgebervertreter agierten erstmals auf internationaler Ebene weisungsungebunden. Bei der Besetzung dieser Funktionen wurde besonders darauf Bedacht genommen, Frauen zu berücksichtigen (Art. 334). Das Internationale Arbeitsamt stand unter der Leitung eines Verwaltungsrates, der aus 24 Mitglieder bestand (Art. 338) und wo sich der Tripartismus fortsetzte: 12 Vertreter der Regierungen ${ }^{92}$, sechs Personen, die von den zur Hauptversammlung abgeordneten Vertretern der Arbeitgeber und sechs

\footnotetext{
${ }^{90}$ StGBl. 303/1920.

${ }^{91}$ RODGERS u.a., International Labour Organization 12.

${ }_{92}$ Davon werden acht durch jene Mitgliedstaaten ernannt, denen die größte industrielle Bedeutung zukommt. Sollte es in dieser Frage "größte industrielle
}

Personen, die von den zur Hauptversammlung abgeordneten Vertretern der Angestellten und Arbeiter gewählt wurden. An der Spitze des Internationalen Arbeitsamts stand ein Leiter, den der Verwaltungsrat ernannte (Art. 339). Dieser empfing vom Verwaltungsrat die Anweisungen und war ihm gegenüber verantwortlich; er nahm an allen Sitzungen des Verwaltungsrates teil, ernannte, ähnlich wie der Generalsekretär des Völkerbundes, das Personal, das Personen verschiedener Staatsangehörigkeiten und Frauen umfassen sollte (Art. 340). Das Internationale Arbeitsamt hatte u.a. die Aufgabe, alle Unterlagen über internationale arbeitsrechtliche Regelungen $\mathrm{zu}$ sammeln, ${ }^{93}$ und wurde als eine weltumspannende Einrichtung zur Erlangung von Auskünften über alle Arbeitsfragen und über die Entwicklung der Arbeitsgesetzgebung betrachtet. ${ }^{44}$ Es arbeitete dahingehend mit dem Völkerbund zusammen, indem es die Mitwirkung des Völkerbund-Generalsekretärs bei Fragen, die den Völkerbund betrafen, in Anspruch nehmen konnte (Art. 343). Jeder Mitgliedstaat konnte gegen jenen Mitgliedstaat, der ein Übereinkommen nicht einhielt, Klage beim Internationalen Arbeitsamt einlegen (Art. 356). Das Internationale Arbeitsamt in Basel existierte noch zur Zeit der Gründung des "ILO-Arbeitsamtes", seine Agenden wurden aber dem Genfer Arbeitsamt übertragen. ${ }^{95}$

Der Generalsekretär hat ebenfalls bei der Zusammensetzung eines Untersuchungsausschusses das Recht, Mitglieder zu bestimmen (Art. 357 Abs. 4). Art. 357-359 beinhalten die Bestimmungen bezüglich der Einsetzung des Untersuchungsausschusses. Es oblag dem Generalsekretär des Völkerbundes das Ergebnis des Untersuchungsausschusses den streitenden Parteien zu übermitteln und er veranlasste dessen Veröffent-

Bedeutung“ zu Streitigkeiten kommen, sollte der Völkerbund entscheiden.

${ }^{93}$ StGBl. 303/1920, Art. 341.

${ }^{94}$ FONTAINE, Internationale Arbeitsorganisation 10.

${ }^{95}$ KUNZ, Internationales Arbeitsrecht 64. 
lichung (Art. 360 Abs. 1); die beteiligten Regierungen mussten binnen einer Frist von einem Monat dem Generalsekretär mitteilen, ob sie das Ergebnis annahmen oder nicht (Art. 360 Abs. 2). Als letzte Streitschlichtungsinstanz galt der Ständige Internationale Gerichtshof (Art. 361), gegen seine Entscheidung war kein Rechtsmittel gegeben (Art. 362). Der Ständige Internationale Gerichtshof konnte etwaige Anträge oder Vorschläge des Untersuchungsausschusses bestätigen, abändern oder aufheben. Er hatte gegebenenfalls die wirtschaftlichen Strafmaßnahmen zu bezeichnen (Art. 363), wozu die Mitgliedstaaten berechtigt waren, sollte ein Mitgliedstaat sich nicht in der gegebenen Frist an die Vorschläge des Untersuchungsausschusses bzw. Entscheidung des Ständigen Internationalen Gerichtshofes (Art. 364) halten. Die „schuldige“ Regierung konnte mit Zustimmung des Generalsekretärs des Völkerbundes einen Untersuchungsausschuss zur Nachprüfung beantragen (Art. 365).

Die Art. 366-368 beinhalteten „Allgemeine Vorschriften“: Jene Mitgliedstaaten, die über Kolonien verfügten, konnten die Übereinkommen in jenen Kolonien, die „keine völlige Selbstregierung haben" dann in Kraft setzen, wenn durch das Abkommen örtliche Verhältnisse nicht verändert wurden (Art. 366). Vertragsabänderungen konnten nur mit der Zweidrittelmehrheit der Anwesenden in der Hauptversammlung angenommen werden. Sie wurden rechtswirksam, sobald sie von drei Viertel der Mitgliedstaaten, die im Völkerbundrat vertreten waren, ratifiziert wurden (Art. 367). Alle Streitfragen oder Schwierigkeiten, die sich durch die Statuten der ILO ergaben, unterlagen der Entscheidung des Ständigen Internationalen Gerichtshofs (Art. 368).

In den „Übergangsbestimmungen“ (Art. 369371) traf man Vorkehrungen für die erste Tagung der ILO, die im Oktober 1919 in Washington

\footnotetext{
${ }^{96}$ MACHACEK, Internationale Arbeitsorganisation 756.

${ }^{97}$ FEHLINGER, Internationale Arbeitsorganisation 1518.

${ }^{98}$ KUNZ, Internationale Arbeitsrecht 65.
}

stattfinden sollte. Die Kosten sollten bis zur Inkraftsetzung des Völkerbundpaktes nach dem Schlüssel des Internationalen Büros des Weltpostvereins festgesetzt werden (Art. 369). Die Regierungen kamen für die Reise- und Aufenthaltskosten ihrer Delegierten auf. Der Leiter des Internationalen Arbeitsamtes übernahm so lange die Funktion des Generalsekretärs des Völkerbundes, bis dieser errichtet sein wird (Art. 370-371). In der Anlage befand sich die Tagesordnung für diese erste Sitzung der ILO: Darunter sind folgende Punkte subsumiert: Durchführung des Grundsatzes des Achtstundentages oder der Achtundvierzigwochenstunde; Fragen hinsichtlich der Verhütung von Arbeitslosigkeit; Beschäftigung der Frauen vor und nach der Niederkunft, Nachtarbeit, gesundheitsschädliche Arbeiten oder Beschäftigung der Kinder in Bezug auf die Altersgrenze, Nachtarbeit und gesundheitsschädlicher Arbeiten.

Das zweite Papier ist mit „Allgemeine Grundsätze" betitelt und diese, die Machacek als "Sozialcharta" bezeichnet, ${ }^{96}$ verpflichteten die Mitgliedstaaten dazu, Arbeit nicht als bloßes Handelsgut zu betrachten sowie zur Umsetzung weiterer arbeitsrechtlicher Bestimmungen wie Vereinigungsfreiheit, 8-Stunden-Tag oder 48-StundenWoche, ein freier Tag pro Woche, Verbot der Kinderarbeit, Einführung des Arbeitsinspektorats sowie zum Grundsatz „gleicher Lohn für gleiche Arbeit ohne Unterschied des Geschlechts" (Art. 372).

Gemäß Art. 369 wurde am 29. Oktober 1919 in Washington die erste Tagung der ILO eröffnet, ${ }^{97}$ Österreich und Deutschland waren nicht vertreten, doch entschied die Hauptversammlung einstimmig die Zulassung von beiden Staaten. ${ }^{98}$ Der offizielle Beitritt Österreichs erfolgte am 28. Mai 1920.99 Auf dieser ersten Sitzung wurde der Fran-

${ }^{99}$ StGB1. 234/1920. 
zose Albert Thomas zum Direktor des Arbeitsamtes gewählt und Genf als Sitz der ILO bestimmt. ${ }^{100}$ Dort arbeiteten 1929400 Beamte aus 36 Nationen.101 Nach Thomas' Tod folgte ihm Harold Butler bis 1938; 1939 wählte man John Gilbert Winant, ihm folgte 1941 Edward Phelan. ${ }^{102}$ Letzterer ging 1948, als die ILO bereits eine UNOrganisation war, in Pension. 1926 rief man ein Expertenkomitee ein, bestehend aus unabhängigen Juristen, das über die Einhaltung der ILOStatuten zu wachen und jährlichen Bericht darüber zu tätigen hatte: Es besteht noch heute. ${ }^{103}$

Auf der ersten Sitzung in Washington wurden sechs Beschlüsse zur Arbeitsplatzsicherheit, Arbeitszeit und zum Schutz von Frauen und Kindern, die in der Industrie angestellt waren, gefasst. ${ }^{104}$ Bereits in den ersten zwei Jahren wurden neun Konventionen erlassen und zehn Empfehlungen angenommen. ${ }^{105}$ Das System der Beschlussfassung - Konventionen und Empfehlungen - bildete damals ein weiteres Unterscheidungsmerkmal zu anderen internationalen Orga-

100 KUNZ, Internationale Arbeitsrecht 64.

${ }^{101}$ FONTAINE, Internationale Arbeitsorganisation 9.

102 JOHNSTON, International Labour Organisation 305306.

${ }^{103}$ https://www.ilo.org/global/about-the-ilo/his-

tory/lang--en/index.htm (Zugriff: 12. 12. 2018)

104 Rosenberg, Transnationale Strömungen 844.

${ }^{105}$ https://www.ilo.org/global/about-the-ilo/history/lang--en/index.htm (Zugriff: 28. 12. 2018): Ihre Inhalte bezogen sich auf Arbeitszeit, Arbeitslosigkeit, Mutterschutz, Nachtarbeit für Frauen, Mindestlohn und Nachtarbeit für Jugendliche.

106 RODGERS u.a., ILO 19: die Britische Regierung trat für Konventionen ein, die US-amerikanische für Empfehlungen, der Kompromiss aus den Verhandlungen war, beide Formen zuzulassen. Konvention sind für die Unterzeichnerstaaten ab dem Zeitpunkt bindend, wenn sie ratifiziert wurden, Empfehlungen müssen innerhalb einer Frist innerstaatlich jenen Stellen unterbreitet werden, um weitere Maßnahmen zu setzen vgl. NeUHOLD, HuMMER, SCHREURER, Österreichisches Handbuch 87.

107 JOHNSTON, International Labour Organisation 310312.

108 Ebd. 103. nisationen, das der Bezeichnung der ILO als Meilenstein rechtfertigt. ${ }^{106}$ Insgesamt wurden während des Zeitraums von 1919 bis 193967 Konvention verabschiedet, ${ }^{107} \mathrm{im}$ selben Zeitraum erfolgte ein rapider Anstieg an Ratifikationen, von 200 im Jahr 1926 über 5001932 bis 800 im Jahr 1938. ${ }^{108}$ Österreich hat während dieser Zeit 13 Konventionen unterschrieben. ${ }^{109}$ Die Konventionen spiegeln die Probleme der damaligen Zeit wider und sind Beweis, dass die ILO auf dem Gebiet des Arbeitsrechts Standards setzte. Die Hauptprobleme jener Zeit betrafen die Bereiche „Arbeitskraft und Beschäftigung“. So etwa nahm sich die ILO der Arbeitslosigkeit an und hat z.B. Studien und Reports für die Ursachen der Arbeitslosigkeit, Wirtschafts- und Sozialpolitik im Laufe der Zwischenkriegszeit in ihrem regelmäßig erscheinenden Journal International Labour Review veröffentlicht. ${ }^{110}$

In der Satzung der ILO werden Menschenrechte im Sinne der Gleichberechtigung formuliert, die während der Zwischenkriegszeit in Konventio-

${ }^{109}$ MACHACEK, Internationale Arbeitsorganisation, folgende Abkommen wurden unterzeichnet: Nr. 1: über die Begrenzung der Arbeitszeit (BGBl. 227/1924); Nr. 2 über die Arbeitslosigkeit (BGBl. 226/1924); Nr. 4 über die Nachtarbeit der Frauen (BGBl. 226/1924); Nr. 5 über das Mindestalter bei Kinderarbeit im Gewerbe (BGBl. 279/1936); Nr. 6 über die Nachtarbeit Jugendlicher (BGB1. 226/1924); Nr. 10 über das Mindestalter bei Kinderarbeit in der Landwirtschaft (BGBl. 226/1924); Nr. 11 über das Kündigungs- und Koalitionsrecht in der Landwirtschaft (BGBl. 226/1924); Nr. 13 über die Anwendung von Bleiweiß zum Anstrich (BGBl. 226/1924); Nr. 17 über die Entschädigung bei Betriebsunfällen (BGBl. 40/1937); Nr. 18 über die Entschädigung bei Berufskrankheiten (BGB1. 278/1936); Nr. 19 über die Gleichbehandlung in der Entschädigung bei Betriebsunfällen (BGBl. 228/1928); Nr. 33 über das Mindestalter bei nichtgewerblicher Kinderarbeit (BGBl. 1936/280); Nr. 45 über Bergarbeit der Frauen (BGBl. 1937/324).

${ }^{110}$ JOHNSTON, International Labour Organisation 135 und RODGERS u.a., ILO 108. 
nen oder Empfehlungen umgesetzt wurden. Unter ihnen befinden sich bahnbrechende Forderungen, die noch heute Gültigkeit haben. Dazu zählen etwa Regelungen bezüglich des Mindestalters in der Industrie auch für Frauen und Kinder, Nachtarbeit für Frauen und Kinder, Assoziationsrecht, Verbot von Kinderarbeit, Mutterschutz oder Mindestlohn im Sinne gleicher Lohn bei gleicher Arbeit.111 Da diese Forderungen in erster Linie die damalige „westliche“ Welt betrafen, erfolgte seitens der ILO zwischen 1930 und 1939 die Differenzierung in ,entwickelte und unterentwickelte Regionen“. Unter diesem Blickwinkel erfolge die Verabschiedung der Konvention Nr. 29 (Verbot der Zwangsarbeit), die in allen Regionen der Welt Gültigkeit besaß und somit auch die indigene Bevölkerung miteinschloss. ${ }^{112}$ Im Zuge dieser Konvention diskutierte man die niedrigeren Anforderungen für Kinder in China, Japan und Indien, die man mit dem Klima, der wirtschaftlichen Entwicklung oder Mangel an Armengesetzen legitimierte. ${ }^{113}$ Auch in Bezug auf Frauenarbeit setzte die ILO wichtige innovative Schritte, wozu sie auf Druck der internationalen Frauenbewegung bewogen wurde. ${ }^{114}$ Abgesehen von der 1906 erlassenen internationalen Konvention des Verbotes von Nachtarbeit für Frauen in der Industrie, erließ die ILO 1919 Konvention Nr. 3 (Mutterschutz) und im selben Jahr Konvention Nr.4 (Nachtarbeitverbot für Frauen), die 1934 novelliert wurde. Empfehlungen beinhalteten etwa die Arbeitsinspektion (Empfehlung Nr. 20, 1923 sowohl Männer als auch Frauen sollten als Arbeitsinspektor/innen eingesetzt werden) oder gleicher Lohn bei gleicher Arbeit für Männer und Frauen (Empfehlung Nr. 30/1928). ${ }^{115}$ Unter Albert Thomas wurde 1932 eine eigene Kommission für Frauenarbeit gegründet, deren Leitung man der französischen

${ }^{111}$ Vgl. dazu JOHNSTON, International Labour Organisation.

112 Dazu ausführlich DAUGHTON, ILO.

${ }^{113}$ RODGERS u.a., ILO 70.

${ }^{114}$ NATChKOVA, SCHOENI, The ILO 49.

115 RODGERS u.a., ILO 57-58.
Feministin Marguerite Thibert übertrug. ${ }^{116}$ Regelungen die Kinderarbeit betreffend wurden in der Konvention Nr. 5 (Mindestalter für Industriearbeit) und Nr. 6 (Nachtarbeit für Jugendliche in der Industrie) von 1919 geregelt - das Mindestalter betrug 14 Jahre. 1932 wurde mit Konvention 33 das Mindestalter auch auf nichtindustrielle Arbeit erweitert. Die ILO konnte zwar der Massenarbeitslosigkeit, die durch die Weltwirtschaftskrise ausgelöst wurde, nicht mittels Konventionen und Empfehlungen Einhalt gebieten, doch hat sie auf dem Gebiet der Wirtschaftsanalysen oder der Suche nach neuen Wirtschaftssystemen gute Arbeit geleistet - alle diese Bemühungen waren Ausdruck des Leitbildes der ILO, dass zwischen internationaler Wirtschaft und Sozialpolitik Kohärenz bestehe; Wirtschafts- und Sozialfragen basieren auf sozialer Gerechtigkeit. ${ }^{117}$

\section{Zusammenfassung}

Der ILO war mehr Erfolg beschieden als dem Völkerbund: 1944 wurde die Philadelphia Charta unter Einbeziehung wichtiger Punkte der Atlantik-Charta angenommen, in der der Grundsatz festgehalten wurde, dass sich alle Menschen unabhängig von Rasse, Geschlecht und Glaubensbekenntnis entfalten können und das Recht auf gleichen Zugang zu materiellem Wohlstand, geistigem Eigentum, Freiheit, Würde und wirtschaftlicher Sicherheit besitzen. ${ }^{118}$ Am 9. Oktober 1946 gab sich die ILO auf der Basis der 1944 formulierten Philadelphia-Konvention eine neue Verfassung und wurde gleichzeitig in die UNO aufgenommen. Im Jahr ihres 50. Geburtstages erhielt die ILO 1969 den Friedensnobelpreis. ${ }^{119}$ Nicht dem Völkerbund, sondern seinem Gründer, Woodrow Wilson, wurde diese Ehre zuteil:

\footnotetext{
116 NATChKOVA, SCHOENI, The ILO 54-60.

117 RODGERS u.a., ILO 207-208

118 KÖCK, Das Recht 428.

${ }^{119}$ RoDGERS u.a., The ILO 2.
} 
1919 verlieh ihm das Friedensnobelpreiskomitee diese hohe Auszeichnung. Wilsons Idee war beseelt, doch konnte er nur wenige seiner 14 Punkte im Völkerbund umsetzen. Dieser scheiterte in erster Linie daran, dass die USA ihm fernblieben, womit das Prinzip der Universalität nicht verwirklicht werden konnte: Der Völkerbund blieb eine weitgehend "eurozentristische Organisation". ${ }^{120}$ Wilson hatte sowohl die Opposition im eigenen Land unterschätzt als auch die Machtpolitik Europas, die in erster Linie von England und Frankreich getragen wurden. Die am Völkerbund vorbei agierende Politik einiger europäischer Staaten trug ebenfalls zum Misserfolg bei wie ein mangelhaftes und rechtlich zahnloses Institutionengefüge. Das Prinzip der kollektiven Sicherheit und jenes der friedlichen Streitbeilegung bot keine wirksamen Maßnahmen gegen die ab 1930 sich häufenden kriegerischen Konflikte und konnten den Ausbruch des Zweiten Weltkrieges nicht verhindern. Gemeinsam mit der Abrüstungsfrage stellen die oben genannten Prinzipien auch gegenwärtig (unlösbare) Bereiche der internationalen Sicherheit dar. ${ }^{121}$

Als integrierender Bestandteil der Friedensverträge erschien der Völkerbund vielen Kritikern als konstitutives Element der Pariser Nachkriegsordnung. Im Nachhinein gesehen war vor allem die Junktimierung mit dem Versailler Vertrag unglücklich, wie auch die verpflichtende Mitgliedschaft der Verliererstaaten, so wurde ihm die volle Anerkennung als erste universelle Friedensorganisation nicht zuteil. Nachhaltigkeit erlangte der Völkerbund in Bezug auf Internationalisierung in ökonomischer, sozialer und kultureller Hinsicht. Seine Organisationsstruktur hatte zukunftsweisenden Charakter, sie wurde in der UNO weiterentwickelt, was auch für den Ständigen Internationalen Gerichtshof gilt. Viele Rechtstheoretiker sehen in ihm den Impulsgeber des institutionalisierten Völkerrechts. ${ }^{122}$

\footnotetext{
${ }^{120}$ UNSER, Die UNO 16.

${ }^{121}$ Vgl. dazu IPSEN, Völkerrecht 1060.

122 TAMS, League of Nations (Zugriff 8. 8. 2018).
}

Als am 24. Oktober 1945 die Charta der Vereinten Nationen in Kraft trat, existierte der Völkerbund noch juristisch. Schließlich fassten am 18. April 194634 Vertreter der Völkerbundmitgliedstaaten den einstimmigen Beschluss, den Völkerbund mit Wirkung 19. April 1946 aufzulösen. Alle Rechte und Pflichten, Besitzungen und Verbindlichkeiten wurden auf die UNO übertragen. ${ }^{123}$ In dieser letzten Versammlung der League beantwortete Lord Robert Cecil eine Frage, die er selbst stellte: „Is it true that all our efforts for those twenty years have been thrown away? For the first time an organisation was constructed, in essence universal, not to protect the national interest of this or that country [...] but to abolish war. The League had been a great experiment."124

\section{Korrespondenz:}

Prof. Dr. Anita ZiEGERHOFER

Universität Graz

Fachbereich Rechtsgeschichte

Institut für rechtswissenschaftliche Grundlagen

Universitätsstraße 15 Bauteil A1

8010 Graz

anita.ziegerhofer@uni-graz.at

ORCID-Nr. 0000-0002-4839-4083
${ }^{123}$ Pfeil, Völkerbund 145.
${ }^{124}$ MAcMilLAN, Peacemakers 84. 


\section{Abkürzungen:}

IALL International Association for Labour Legislation ILO International Labour Organization

Siehe auch das allgemeine Abkürzungsverzeichnis: [http://www.rechtsgeschichte.at/files/abk.pdf]

\section{Literatur:}

Winfried BAUMGART, Vom europäischen Konzert zum Völkerbund. Friedensschlüsse und Friedenssicherung von Wien bis Versailles (= Erträge zur Forschung 25, Darmstadt 21987).

Winfried BÖTTCHER (Hg.) Klassiker des europäischen Denkens. Friedens- und Europavorstellungen aus 700 Jahren europäischer Kulturgeschichte (BadenBaden 2014).

Paul BARANDON, Völkerbund, in: SCHLOCHAUER, Wörterbuch des Völkerrechts 3, 597-611.

Richard Coudenhove-Kalergi, Pan-Europa und der Völkerbund, in: Pan-Europa 6 (1924) 3-23.

DERS., Weltpolitik, in: Pan-Europa 8/10 (1925) 32-37.

Paul ECKHARDT, Ewald KUTTIG, Das international Arbeitsrecht im Friedensvertrage. Kommentar zum Teil XIII des Friedensvertrages von Versailles (Berlin 1920) (Veröffentlichung aus dem Kommentar zum Friedensvertrag hg. v. Walter SCHÜCKING).

J.P. DAUGHTON, ILO Expertise and Colonial Violence in the Interwar Years, in: Sandrine KотT, Joelle Droux, Globalizing Social Rights. The International Labour Organization and Beyond (Basingstoke 2013) 85-97.

H. FEHLINGER, Die internationale Arbeitsorganisation und ihr Wirken (Leipzig 1922).

Arthur FONTAINE, in: Internationales Arbeitsamt (Hg.), Die Internationale Arbeitsorganisation 1919-1929 (Genf 1930) 7-14.

Rudolf FREUND, Die Genfer Protokolle. Ihre Geschichte und Bedeutung im Staatsleben Deutsch-Österreichs (= Sozialwissenschaftliche Forschungen V/2, Berlin-Leipzig 1924).

Hermann FRIEDMANN, Vom Völkerbund zur UNO. Wege internationaler Verständigung (Gerabronn 1953).

Otto GÖPPERT, Der Völkerbund. Organisation und Tätigkeit des Völkerbundes (Stuttgart 1938).

Erik GOLDSTEIN, The First World War Peace Settlements, 1919-1925 (London 2002).

Jürgen HeIDEKING, Oberster Rat - Botschafterrat - Völkerbund. Drei Formen multilateraler Diplomatie nach dem Ersten Weltkrieg, in: HZ 231 (1980) 589_ 630.

Hans Joachim HeISER, Abrüstung, in: SCHLOCHAUER, Wörterbuch des Völkerrechts 1, 5-9.

Ruth Beatrice HENIG, The League of Nations (Edinburgh 1973).

Madeleine HERREN, Internationale Organisationen seit 1865. Eine Globalgeschichte der internationalen Ordnung (Darmstadt 2009).

Martyn HousDen, The League of Nations and the Organisation of Peace (Harlow 2012).

Ernst Rudolf HuBER, Deutsche Verfassungsgeschichte seit 1789, Bd. 7: Ausbau, Schutz und Untergang der Weimarer Republik (Stuttgart u.a. 1984).

Internationales Arbeitsamt (Hg.), Zehn Jahre Internationale Arbeitsorganisation (Genf 1931).

Internationales Arbeitsamt (Hg.), Die internationale Arbeitsorganisation 1919-1929 (Genf 1930).

Arika IRIYe, Jürgen Osterhammel (Hgg.), Geschichte der Welt 1870-1945 (München 2012).

George A. JOHNSTON, The International Labour Organisation. It's work for social and economic progress (London 1970).

Ian KERSHAW, Hitler 1889-1936 (Stuttgart 1998).

Herbert Franz KÖCK, Peter FISCHER, Das Recht der Internationalen Organisationen (Wien ${ }^{3}$ 1997).

Sandrine KOTT, Joelle Droux, Globalizing Social Rights. The International Labour Organization and Beyond (Basingstoke 2013).

Josef KunZ, Das internationale Arbeitsrecht der Pariser Friedensverträge und seine Entwicklung, in: Zeitschrift für öffentliches Recht 2/3-6 (1921) 39-69.

Jörn LEONHARD, Der überforderte Frieden. Versailles und die Welt 1923 (München 2018).

Rudolf MACHACEK, Die Internationale Arbeitsorganisation (International Labour Organisation - ILO), in: Oswin MARTINEK (Hg.), Arbeit, Recht und Gesellschaft. Festschrift Walter Schwarz zum 65. Geburtstag (Wien 1991) 753-767.

Margaret MACMiLlan, Die Friedensmacher. Wie der Versailler Vertrag die Welt veränderte (Berlin 2018).

Margaret MaCmILLAN, Peacemakers. Six Months that Changed the World (London 2001).

Erez MANELA, The Wilsonian Moment. Self-Determination and the International Origins of Anticolonial Nationalism (New York 2007).

Oswin MARTINEK (Hg.), Arbeit, Recht und Gesellschaft. Festschrift Walter Schwarz zum 65. Geburtstag (Wien 1991). 
Nora NAtchKova, Céline SchOENI, The ILO, Feminists and Experts Networks: The Challenges of a Protective Policy (1919-1934), in: Sandrine KoTT, Joelle Droux, Globalizing Social Rights. The International Labour Organization and Beyond (Basingstoke 2013) 49-64.

Hanspeter NeuHOLD, Waldemar HuMMeR, Christoph SCHREUER (Hgg.), Österreichisches Handbuch des Völkerrechts, Bd. 1: Textteil (Wien 2004).

Gino OLIVETTI, Zehn Jahre Tätigkeit, in: Internationales Arbeitsamt, Die internationale Arbeitsorganisation $15-22$.

Garry B. OstrowER, The League of Nations from 1919 to 1929 (New York 1996).

Clive PARRY, League of Nations, in: Rudolf BERNHARDT (Hg.), Encyclopedia of public International Law, Bd. III (Amsterdam 1997) 177-186.

Susan Pedersen, The Meaning of the Mandates System: An Argument, in: Geschichte und Gesellschaft 32 (2006) 560-582.

Alfred PFEIL, Der Völkerbund (= Erträge der Forschung 58, Darmstadt 1976).

Walter PoEgGeL, Der Völkerbund (Bonn 1995).

Sarah PRITCHARD, Der völkerrechtliche Minderheitenschutz. Historische und neue Entwicklungen, (= Tübinger Schriften zum internationalen und europäischen Recht 55, Berlin 2001).

Laura RATHMANNER, Die Reparationskommission nach dem Staatsvertrag von St. Germain, in: BRGÖ 6 (2016) 74-98.

Kurt von RAUMER, Rudolf VIERHAUS, Friede und Völkerordnung Teil II: Friedensbewegung und internationale Organisation (Stuttgart 1971).

Volker RitTBERGER, Bernhard ZANGL, Andreas KRUCK, Internationale Organisationen (Wiesbaden ${ }^{42013) .}$

Gerry RoDGERS u.a., The International Labour Organization and the quest for social justice, 1919-2009 (Geneva 2009).

Emily S. ROSENBERG, Transnationale Strömungen in einer Welt, die zusammenrückt, in: Arika IRIYE, Jürgen Osterhammel (Hgg.), Geschichte der Welt 1870-1945 (München 2012) 815-998.

Hans-Jürgen SCHLOCHAUER, Wörterbuch des Völkerrechts 4 Bde. (Berlin 1960-1962).
Walther SCHÜCKING, Hans WeHBERG, Die Satzung des Völkerbundes (Berlin ${ }^{2} 1924$ ).

Klaus SCHWABE, Weltmacht und Weltordnung. Amerikanische Außenpolitik von 1898 bis zur Gegenwart. Eine Jahrhundertgeschichte (Paderborn 2007).

Reinhard Stauber, Der Wiener Kongress (Wien 2014).

Christian J. TAMS, League of Nations (2007). Max Planck Encyclopedia of Public International Law (Oxford 2007).

Michael THÖNDL, Mussolinis ostafrikanisches Imperium in den Aufzeichnungen und Berichten des deutschen Generalkonsulats in Addis Abeba (1936-1941), in: Quellen und Forschungen aus italienischen Archiven und Bibliotheken (QFIAB) 88 (2008) 449-488.

Erwin VIEFHAUS, Die Minderheitenfrage und die Entstehung der Minderheitenschutzverträge auf der Pariser Friedenskonferenz (= Marburger Ostforschungen 11, Würzburg 1960).

Hans WeHBERG, Briand-Kellogg-Pakt von 1928, in: SCHLOCHAUER, Wörterbuch des Völkerrechts I, 249-250.

Peter YEARWOOD, Guarantee of peace: the League of Nations in British policy, 1914-1925 (Oxford 2009).

Alfred ZIMMERN, The League of Nations and the Rule of Law 1918-1935 (London 1945).

Anita ZIEGERHOFER, League of Nations, in: Ute DANIEL u.a (Hgg.), 1914-1918-online. International Encyclopedia of the First World War (Freie Universität Berlin) https:/encyclopedia.1914-1918-online.net/ article/league_of_nations

\section{Internetquellen:}

http://www.austlii.edu.au/au/other/dfat/treaties/1920/5.html (Zugriff: 12.12.2018)

http://www.austlii.edu.au/au/other/dfat/treaties/1920/14.html (Zugriff: 12.12.2018)

https://www.ilo.org/global/about-the-ilo/history/lang-en/index.htm (Zugriff: 12.12.2018)

https://www.ilo.org/global/about-the-ilo/history/lang-en/index.htm (Zugriff: 28.12.2018) 